Recepción: 20 / 04 / 2017

Aceptación: 20 / 05 / 2017

Publicación: 15 / 06 / 2017
Ciencias de la Comunicación

Artículo de Investigación

\title{
La cultura artística como parte del perfil del docente de la Facultad Comunicación Social de la Universidad de Guayaquil y propuesta de un conjunto de talleres de incentivación y desarrollo de las actividades artísticas
}

The artistic culture as part of the profile of the teacher of the Faculty of Social Communication of the University of Guayaquil and proposal of a set of workshops to encourage and develop artistic activities

\section{Cultura artística como parte do corpo docente da escola de comunicação social da} Universidade de Guayaquil e propôs uma série de oficinas de incentivo e desenvolvimento do perfil de atividades artísticas

\author{
Juan C. Loaiza-Mina \\ juan.loaizam@ug.edu.ec \\ Roberto A. Loayza-Mina \\ roberto.loayzam@ug.edu.ec \\ Lucrecia de las M. Benítes-Mora \\ lucrecia.benitem@ug.edu.ec \\ Johnson Y. Ching-Chiy ${ }^{\mathrm{IV}}$ \\ johnson.chingc@ug.edu.ec
}

Correspondencia: juan.loaizam@ug.edu.ec

Docente Contratado de la Facultad de Arquitectura y Urbanismo de la Universidad de Guayaquil. Gestor de Acreditación de la Facultad de Arquitectura y Urbanismo, Guayaquil, Ecuador.

II. Docente Contratado de la Facultad de Arquitectura y Urbanismo de la Universidad de Guayaquil. Gestor de Acreditación de la Facultad de Arquitectura y Urbanismo, Guayaquil, Ecuador.

III. Docente Contratado de la Facultad de Arquitectura y Urbanismo de la Universidad de Guayaquil. Gestora de Bienestar Estudiantil de la Facultad de Arquitectura y Urbanismo, Guayaquil, Ecuador.

Iv. Docente Contratado de la Facultad de Arquitectura y Urbanismo de la Universidad de Guayaquil. Gestor Académico de la Carrera de Diseño de Interiores de la Facultad de Arquitectura y Urbanismo, Guayaquil, Ecuador. 
Juan C. Loaiza-Mina; Roberto A. Loayza-Mina; Lucrecia de las M. Benítes-Mora; Johnson Y. Ching-Chiy

\section{Resumen}

En el presente proyecto se busca en analizar acerca de la cultura artística que se incluye en el proceso enseñanza aprendizaje del docente en la Universidad de Guayaquil. En este documento se presentan los aspectos más relevantes en relación de la aplicación de la cultura artística, en el marco teórico se encuentran los aportes de investigadores en cultura artística como parte del perfil en docencia superior, el objetivo básicamente es contribuir con el mejoramiento de la calidad educativa de la Universidad de Guayaquil, en donde se incluya la cultura artística. En la metodología aplicada es la investiga descriptiva modalidad de proyecto factible, así como también se considera la aplicación de una investigación de campo. El presente trabajo de grado tiene 3 variables la cultura artística como parte del perfil del docente, incentivación y desarrollo de las actividades artísticas al Docente en la facultad de Comunicación Social en la Universidad de Guayaquil.

Palabras clave: Arte; andragogía; cultura artística; taller de capacitación. 


\begin{abstract}
In the present project, we look for to analyze about the artistic culture that is included in the teaching process of the teacher in the University of Guayaquil. This document presents the most relevant aspects related to the application of artistic culture, in the theoretical framework are the contributions of researchers in artistic culture as part of the profile in higher education, the objective is basically to contribute to the improvement of Educational quality of the University of Guayaquil, where artistic culture is included. In the applied methodology is the investigates descriptive feasible project modality, as well as the application of a field investigation is considered. The present work of degree has 3 variables the artistic culture as part of the profile of the teacher, incentivation and development of the artistic activities to the Teacher in the Faculty of Social Communication in the University of Guayaquil.
\end{abstract}

Key words: Art; andragogy; artistic culture; training workshop. 
Juan C. Loaiza-Mina; Roberto A. Loayza-Mina; Lucrecia de las M. Benítes-Mora; Johnson Y. Ching-Chiy

\section{Resumo}

Neste projeto pretende analisar sobre a cultura artística que está incluído no processo ensinoaprendizagem do ensino na Universidade de Guayaquil. Este documento descreve os aspectos mais relevantes são apresentadas em relação à aplicação da cultura artística no referencial teórico as contribuições de pesquisadores encontrou na cultura artística como parte do perfil no ensino superior, o objetivo é, basicamente, para contribuir para melhorar a educação de qualidade na Universidade de Guayaquil, onde a cultura artística está incluído. Na metodologia é descritiva investiga modalidade de projecto viável, bem como considerando a aplicação de uma investigação de campo. Este trabalho grau tem 3 variáveis cultura artística como parte do ensino, incentivos e o desenvolvimento de atividades artísticas ensino na Faculdade de Comunicação Social no perfil Universidade de Guayaquil.

Palavras-chave: Arte; andragogia; cultura artística; oficina de formação. 


\section{Introducción.}

La enseñanza de la cultura artística motiva el desarrollo de la parte emocional y creativa del ser humano, si se considera que generalmente la pedagogía muestra una conexión que demuestra que la práctica de actividades artísticas contribuye al desarrollo de habilidades de comunicación y el desarrollo físico-emocional cognitivo del individuo, además del hecho de ayuda a mejorar las capacidades expresivas para promover el aprendizaje de la parte lógica.(García Retana, 2012)(Rico, 2009)(Mitchel, 2009)

El presente trabajo se encuentra orientado a analizar el nivel de conocimiento, acerca de la cultura artística por parte de los docentes de la Facultad de Comunicación Social de la Universidad de Guayaquil, considerando que a través de una pre investigación se ha podido identificar que pocos docentes de esta facultad poseen una cultura artística ya que generalmente se han enfocado mucho más en el desarrollo lógico. (Vázquez, Gómez, \& Lugo, 2008)(Yuni \& Urbano, 2010)

Los docentes de la Facultad de Comunicación Social como educadores de las carreras de Publicidad, Periodismo, Turismo, y Diseño, deben poseer una cultura artística, considerando que especialmente en estas carreras el arte se encuentra implícito en todo su contexto debido a que contribuye al desarrollo de las habilidades de comunicación. La estructuración del trabajo se presenta de la siguiente manera:

Se analiza adecuadamente todos los aspectos relacionados al problema de investigación, se identifica la ubicación del problema y se determinan los objetivos de investigación. Además se presenta la correspondiente justificación para el desarrollo de la investigación. 
Juan C. Loaiza-Mina; Roberto A. Loayza-Mina; Lucrecia de las M. Benítes-Mora; Johnson Y. Ching-Chiy

Se desarrolla el marco teórico en dónde se analizan diferentes teorías relacionadas con la cultura artística y los beneficios de que sea aplicada en docencia.

Se incluye la definición de la metodología de investigación, en donde se determina el tipo de investigación que será aplicada y se define la población en base a la cual se desarrollará la investigación.

La tabulación y el análisis de los datos que se obtuvieron del desarrollo de la investigación, los mismos que se presentarán tanto de forma cuantitativa a través de gráficos estadísticos, como de forma cualitativa mediante su respectivo análisis.

\section{Materiales y métodos.}

En la presente investigación se determina la aplicación de un diseño de investigación cuantitativa ya que se busca evaluar el nivel de cultura artística que poseen los docentes de la Facultad de Comunicación Social de la Universidad de Guayaquil, a fin de determinar la necesidad de desarrollar actividades orientadas a promover esta cultura para que el docente desarrolle sus destrezas y habilidades en las actividades artísticas.

Se busca diagnosticar las falencias culturales relacionadas a las artes en los docentes cuyos efectos repercuten directamente en la calidad de la enseñanza. Además de la investigación cuantitativa se determina la aplicación de un método inductivo, considerando lo que determina Goetz y LeCompte (2008), "La investigación puramente inductiva empieza con la recogida de datos, mediante observación empírica o mediciones de alguna clase, y a continuación construye, a partir de las relaciones descubiertas, sus categorías y proposiciones teóricas”. (pág. 30) 
Se determina la aplicación del método inductivo considerando que se parte de un estudio particular a una muestra de docentes de la Facultad de Comunicación Social de la Universidad de Guayaquil para analizar el nivel de cultura artística que poseen, se considerará una muestra representativa del total de docentes de esta facultad de modo que se pueda generalizar y aplicar una propuesta orientada a desarrollar las habilidades y destrezas.

Deductivo: porque se parte de conceptos referentes al desarrollo de habilidades y destrezas en la educación superior, relacionando estos conocimientos con los casos particulares estudiados.

Inductivos: porque partiendo de las observaciones particulares en cuanto a las habilidades y destrezas que se fundamentan en la teoría existente, en principios o leyes, de los que se vale para dar explicación razonada a los supuestos.

Respecto al método deductivo, este es aquel que se fundamenta en la teoría existente, en principios o leyes, de los que se vale para dar explicación razonada a los supuestos.

Para la aplicación del método deductivo, el proyecto se sustentó en las teorías existentes acerca de las artes plásticas, como componente del área de cultura estética.

En cuanto al método inductivo, este es un proceso de razonamiento lógico que se inicia con la recopilación de datos estos son separados en variables que se jerarquizan y categorizan, y finalmente, se establecen comparaciones de características, propiedades y relaciones funcionales entre los datos de las observaciones. Su aplicación en el presente proyecto ha seguido su característico camino de lo particular a lo general, a través de las siguientes fases: observación, comparación. Abstracción y generalización. 
Juan C. Loaiza-Mina; Roberto A. Loayza-Mina; Lucrecia de las M. Benítes-Mora; Johnson Y. Ching-Chiy

\section{Modalidad de la investigación}

El presente proyecto de investigación se desarrolla bajo la modalidad de campo, dado que se efectúa en el mismo lugar donde se ha detectado el problema, esto es la Facultad de comunicación social de la UG. Esto ha permitido obtener información de primera mano y en forma directa.

En cuanto a los objetivos que se persigue, es de modalidad aplicada, pues se sirve de los adelantos de la investigación superior y le interesa su aplicación inmediata en el desarrollo de habilidades de expresión plástica en la Facultad objeto de estudio. Para Cohen y Manían este tipo de investigación es adecuada "siempre que se requiera un acontecimiento" específico para un problema específico en una situación específica.

\section{Tipo de investigación}

La investigación tendrá la siguiente tipología:

Por la naturaleza será de tipo exploratorio y descriptivo.

$\checkmark$ En un primer nivel, y dada su naturaleza, será de tipo Exploratorio, porque se efectuará un sondeo sobre la aplicación de técnicas plásticas por parte del docente.

$\checkmark$ En un segundo nivel, será descriptivo, porque consistirá en describir las situaciones que se dan en el aula para propiciar el desarrollo de habilidades artísticas de alumnas y alumnos, especialmente en lo relacionado a las artes plásticas.

En un tercer nivel será Explicativo, en vista de que efectuará un análisis de las causas del problema de la no aplicación de técnicas en las artes plásticas que favorezcan el desarrollo de habilidades de expresión, así como las consecuencias derivadas de tal situación.

\section{Población y muestra}




\section{Población}

Está constituida por los docentes, en un total de 78:

\section{Muestra}

Se realizó 65 encuestas.

La muestra la constituyen los docentes de la facultad, quienes serán objeto de la aplicación de encuestas.

Una vez conocido el tamaño de la muestra, la metodología continuará con la aplicación de las técnicas necesarias para la consecución de los objetivos de investigación que se han establecido anteriormente.

Con esto se busca contribuir al desarrollo de los conocimientos científicos en el área de cultura artística, dentro del nivel superior.

Cada uno de los niveles señalados constituye un peldaño que se deberá escalar en la tarea de investigación a fin de obtener poco a poco una mayor identificación con los diferentes aspectos relacionados con el objeto investigado, esto es la importancia de que la cultura artística se de cómo conocimiento general a los docentes ejecutándolos en los talleres de actividades artísticas en la educación superior, particularmente en el desarrollo de habilidades de expresión a través de las arte plásticas y las otras artes representativas, etc.

Además, por su aplicación será factible, dado que será fácil aplicar los instrumentos para la recolección de datos que permitan la confrontación de las hipótesis formuladas.

Instrumentos de la investigación 
Juan C. Loaiza-Mina; Roberto A. Loayza-Mina; Lucrecia de las M. Benítes-Mora; Johnson Y. Ching-Chiy

Como instrumentos de investigación, está el cuestionario, el cual fue esencial para conocer la aceptación por parte de los docentes, para desarrollar el taller de artes plásticas

\section{Resultados.}

Análisis e interpretación de datos

¿Para usted el arte es una opción que se puede implementar en los centros educativos para enriquecer y fomentar la cultura del país?

\begin{tabular}{|l|l|l|l|}
\hline \multirow{4}{*}{$\begin{array}{l}\text { ÍTEM } \\
\mathbf{1}\end{array}$} & Valor & Frecuencia & Porcentaje \\
\cline { 2 - 4 } & Dotal acuerdo & 32 & $49 \%$ \\
\cline { 2 - 4 } & $\begin{array}{l}\text { Ni de acuerdo } \\
\text { desacuerdo ni }\end{array}$ & 15 & $23 \%$ \\
\cline { 2 - 4 } & Desacuerdo & 10 & $5 \%$ \\
\cline { 2 - 4 } & $\begin{array}{l}\text { Total } \\
\text { Desacuerdo }\end{array}$ & 5 & $15 \%$ \\
\cline { 2 - 4 } & TOTAL & 65 & $8 \%$ \\
\hline
\end{tabular}

\section{Cuadro 1.- Consideran el arte como una opción}

Para el 49\% de la población encuestada está de acuerdo con que la integración de las artes puede enriquecer la cultura y fomentarla en la juventud, mientras que el $23 \%$ esta solo de acuerdo, el $5 \%$ se muestra indiferente, un $15 \%$ manifiesta su desacuerdo respecto a esta propuesta planteada y solamente un $8 \%$ está en total desacuerdo con la propuesta. Lo que quiere decir que los docentes consideran en su mayoría que hay factibilidad en esta idea.

2.- ¿Qué grado de conocimiento considera tener en el campo de las artes?

\begin{tabular}{|l|l|l|l|}
\hline \multirow{2}{*}{$\mathbf{2}$} & Valor & Frecuencia & Porcentaje \\
\cline { 2 - 4 } & Insuficiente & 37 & $57 \%$ \\
\cline { 2 - 4 } & Poco & 15 & $23 \%$ \\
\cline { 2 - 4 } & Moderado & 3 & $5 \%$ \\
\cline { 2 - 4 } & Necesario & 10 & $15 \%$ \\
\hline
\end{tabular}




\begin{tabular}{|l|l|l|l|}
\hline & Total & 65 & $100 \%$ \\
\hline
\end{tabular}

\section{Cuadro 2.- Grado de conocimientos de docentes en arte}

Análisis: Los docentes fueron interrogados respecto al grado de conocimiento que tienen según su apreciación propia y la encuesta arrojo las siguientes respuestas, el 57\% de los encuestados considera que sus conocimientos en el campo de las artes son insuficientes, seguido del $23 \%$ que dice tener poco conocimiento, el 5\% tiene moderados conocimientos y solamente un 15\% manifestó tener los conocimientos que se requieren para impartir la enseñanza en el campo de las artes a los estudiantes.

3.- ¿Cuál o cuáles de estas disciplinas artísticas, cree que se puedan implementar en el pensum educativo de los estudiantes?

\begin{tabular}{|l|l|l|l|}
\hline & Valor & Frecuencia & Porcentaje \\
\hline \multirow{4}{*}{$\mathbf{3}$ TEM } & Arquitectura & 3 & $5 \%$ \\
\cline { 2 - 4 } & Música & 30 & $46 \%$ \\
\cline { 2 - 4 } & Danza & 8 & $12 \%$ \\
\cline { 2 - 4 } & Cine & 14 & $22 \%$ \\
\cline { 2 - 4 } & Literatura & 2 & $3 \%$ \\
\cline { 2 - 4 } & Pintura & 7 & $11 \%$ \\
\cline { 2 - 4 } & Escultura & 1 & $2 \%$ \\
\cline { 2 - 4 } & Total & 65 & $100 \%$ \\
\hline
\end{tabular}

\section{Cuadro 3.- Disciplinas artísticas que pueden implementarse}

Análisis: El 46\% de los docentes considera que la música es una buena opción entre las artes que se puede implementar en la facultad, a esto le sigue con un $22 \%$ Cine, ya que se considera que tendrá un gran aporte profesional para los estudiantes de todas las carreras que existen en la Facso, el $12 \%$ indica que prefiere danza, mientras que el $11 \%$ nombra a pintura como la más idónea y finalmente se ubican con un 3\% arquitectura y con solo un $1 \%$ la escultura siendo el arte que menor grado de aceptación tuvo en la encuesta realizada. 
Juan C. Loaiza-Mina; Roberto A. Loayza-Mina; Lucrecia de las M. Benítes-Mora; Johnson Y. Ching-Chiy

4.- ¿Según su criterio, cuál de estas razones es la que mayormente ha impedido realizar un programa que integre al arte en centros educativos?

\begin{tabular}{|c|c|c|c|}
\hline & Valor & Frecuencia & Porcentaje \\
\hline & $\begin{array}{l}\text { Falta de } \\
\text { interés por } \\
\text { parte }\end{array}$ & & \\
\hline & $\begin{array}{l}\text { del } \\
\text { gobierno }\end{array}$ & 25 & $38 \%$ \\
\hline & $\begin{array}{l}\text { Interés } \\
\text { escaso por } \\
\text { el }\end{array}$ & & \\
\hline $\begin{array}{l}\text { ÍTEM } \\
4\end{array}$ & $\begin{array}{l}\text { parte } \\
\text { estudiantes }\end{array}$ & 12 & $18 \%$ \\
\hline & $\begin{array}{l}\text { Poco o nulo } \\
\text { interés por } \\
\text { el arte en } \\
\text { los } \\
\text { docentes }\end{array}$ & 10 & $15 \%$ \\
\hline & $\begin{array}{l}\text { Falta de } \\
\text { personal } \\
\text { capacitado } \\
\text { en el país }\end{array}$ & 18 & $28 \%$ \\
\hline & Total & 65 & $100 \%$ \\
\hline
\end{tabular}

\section{Cuadro 4.-Razones por las que no se ha implementado este proyecto}

Análisis: Entre las razones por las cuales no se ha implementado un proyecto de este tipo tenemos que: el $39 \%$ considera que ha sido debido a la falta de interés por parte del gobierno nacional para implementar al arte en los centros de educación superior. El 28\% cree que esto se debe a la falta de talento humano capacitado respecto al arte y sus disciplinas, ya que no son mayoría personas en el país que se dedican a estudiar carreras artísticas, el 18\% piensa que esta situación tiene que ver con al poco interés que manifiestan los estudiantes por el arte y solamente un $15 \%$ cree que una de las razones es la falta de interés por parte de los docentes de la facultad. 
5.- ¿Cree usted que la ejecución de disciplinas artísticas como materias de estudio contribuirán al desarrollo personal y profesional de los estudiantes?

\begin{tabular}{|l|l|l|l|}
\hline Valor & Frecuencia & Porcentaje \\
\hline Total acuerdo & 42 & $65 \%$ \\
\hline ÍTEM & De acuerdo & 20 & $31 \%$ \\
\hline $\mathbf{5}$ & Ni de acuerdo, ni & & \\
desacuerdo & 3 & $5 \%$ \\
\hline & Desacuerdo & 0 & $0 \%$ \\
\hline Total Desacuerdo & 0 & $0 \%$ \\
\hline
\end{tabular}

\section{Cuadro 5.- Contribución en lo personal y profesional a los estudiantes}

Análisis: En cuanto a las opiniones acerca de la contribución que representa para los estudiantes la implementación de la educación artística tenemos a un $65 \%$ que se pronuncia totalmente de acuerdo, un 31\% está de acuerdo, el 5\% de los docentes manifiesta que no está de acuerdo ni tampoco en desacuerdo en este aspecto y finalmente ninguno de los encuestados manifestó estar en desacuerdo ni en total desacuerdo.

6.- ¿Considera necesario que se dicte un seminario para capacitar a los docentes de la facultad en el campo de las artes?

\begin{tabular}{|l|l|l|l|}
\hline Valor & Frecuencia & Porcentaje \\
\hline Muy necesario & 34 & $52 \%$ \\
\hline ÍTEM & 19 & $29 \%$ \\
\hline $\begin{array}{l}\text { Ni muy importante, } \\
\text { ni } \\
\text { necesario }\end{array}$ & 12 & \\
\hline
\end{tabular}


Juan C. Loaiza-Mina; Roberto A. Loayza-Mina; Lucrecia de las M. Benítes-Mora; Johnson Y. Ching-Chiy

6

\begin{tabular}{|l|l|l|}
\hline Poco necesario & 0 & $0 \%$ \\
\hline Nada necesario & 0 & $0 \%$ \\
\hline Total & 65 & $100 \%$ \\
\hline
\end{tabular}

\section{Cuadro 6.- Necesidad de realizar el taller para docentes}

Análisis: Entre los docentes que participaron de la encuesta el 52\% considera que es muy necesario que se realicen talleres de capacitación para los catedráticos en el campo de artes, ya que consideran que tienen falencias en cuanto a conocimientos en esta área, el $29 \%$ cree que es necesario, solo el 19\% muestra una actitud desinteresada y entre los encuestados ninguno piensa que sea poco necesario, ni tampoco creen que la medida es para nada necesaria.

7.- De los siguientes temas ¿Cuál o cuáles temas le gustaría que se traten en los talleres?

\begin{tabular}{|l|l|l|l|}
\hline & Valor & Frecuencia & Porcentaje \\
\hline \multirow{4}{*}{$\mathbf{8}$ 8.M } & Arquitectura & 1 & $2 \%$ \\
\cline { 2 - 4 } & Música & 17 & $26 \%$ \\
\cline { 2 - 4 } & Danza & 8 & $12 \%$ \\
\cline { 2 - 4 } & Cine & 14 & $22 \%$ \\
\cline { 2 - 4 } & Literatura & 12 & $18 \%$ \\
\cline { 2 - 4 } & Pintura & 11 & $17 \%$ \\
\cline { 2 - 4 } & Escultura & 2 & $3 \%$ \\
\cline { 2 - 4 } & Total & 65 & $100 \%$ \\
\hline
\end{tabular}

\section{Cuadro 7.- Áreas en las que los docentes quieren ser capacitados}

Análisis: Las disciplinas artísticas en las que destacan interés los maestros encuestados, para los talleres de capacitación son las siguientes: el 26\% prefiere la música, el 22\% el cine, mientras que literatura tiene un $18 \%$ de interés por parte de los docentes, seguida de pintura que abarca un $17 \%$, luego tenemos con un $12 \%$ a danza y con los menores porcentajes de expectativa por parte del personal docente, finalmente se ubican con un $3 \%$ escultura y por ultimo con un $2 \%$ arquitectura.

8.- ¿Está usted dispuesto a asistir a dichos talleres? 


\begin{tabular}{|l|l|l|l|}
\hline \multicolumn{1}{|c|}{$\begin{array}{l}\text { ITEM } \\
\mathbf{8}\end{array}$} & Valor & Frecuencia & Porcentaje \\
\cline { 2 - 4 } & Total acuerdo & 35 & $54 \%$ \\
\cline { 2 - 4 } & $\begin{array}{l}\text { De acuerdo } \\
\text { Ni de acuerdo, } \\
\text { ni desacuerdo }\end{array}$ & 12 & $18 \%$ \\
\cline { 2 - 4 } & Desacuerdo & 5 & $15 \%$ \\
\cline { 2 - 4 } & $\begin{array}{l}\text { Total } \\
\text { Desacuerdo }\end{array}$ & 3 & $8 \%$ \\
\cline { 2 - 4 } & Total & 65 & $5 \%$ \\
\hline
\end{tabular}

\section{Cuadro 8.- Esta dispuesto a asistir a los talleres}

Análisis: Otro dato relevante que permitió dar a conocer la encuesta fue, si el personal considerado para las capacitaciones está dispuesto a asistir a las mismas; en respuesta a esta inquietud tenemos las siguientes variables: el 54\% se encuentra en acuerdo total, le sigue un $18 \%$ que manifiesta que de realizarse dichos talleres está dispuesto a asistir, un $15 \%$ dice que no está ni a favor ni en contra de la mencionada medida y solo un $8 \%$ se pronuncia en desacuerdo y finalmente existe únicamente un 5\% que se opone rotundamente a esta medida por considerarla innecesaria.

9.- ¿En qué jornada le gustaría que se dicten los talleres de arte?

\begin{tabular}{|l|l|l|l|}
\hline & Valor & Frecuencia & Porcentaje \\
\hline \multirow{3}{*}{$\mathbf{9}$} & Mañana & 10 & $15 \%$ \\
\cline { 2 - 4 } & Tarde & 32 & $49 \%$ \\
\cline { 2 - 4 } & Noche & 23 & $35 \%$ \\
\cline { 2 - 4 } & Total & 65 & $100 \%$ \\
\hline
\end{tabular}

\section{Cuadro 9.- Horario que prefieren los docentes para recibir los talleres}

Análisis: En cuanto a los horarios a considerarse para la realización de los talleres se les brindo tres opciones, un $15 \%$ manifiesta que de preferencia les gustaría recibir los talleres en la 
Juan C. Loaiza-Mina; Roberto A. Loayza-Mina; Lucrecia de las M. Benítes-Mora; Johnson Y. Ching-Chiy

jornada de la mañana, a esto le sigue un 36\% que indica que prefiere que la capacitación sea en jornada nocturna y un $49 \%$ indica que puede asistir en la jornada de la tarde, ya que esta le permite laboral con normalidad y sin interrupciones en su horario de trabajo, cabe destacar que este es el grupo que mayor aceptación presenta en cuanto a la horario de preferencia por parte de los docentes y es el que será considerado para su posterior aplicación.

10.- ¿Considera que estos talleres pueden llevar al país a realzar su incursión tanto a nivel nacional como internacional en el campo artístico?

\begin{tabular}{|l|l|l|l|}
\hline & Valor & Frecuencia & Porcentaje \\
\hline \multirow{4}{*}{$\begin{array}{l}\text { 10M } \\
\text { To }\end{array}$} & $\begin{array}{l}\text { Total } \\
\text { acuerdo }\end{array}$ & 35 & $54 \%$ \\
\cline { 2 - 4 } & De acuerdo & 15 & $23 \%$ \\
\cline { 2 - 4 } & $\begin{array}{l}\text { Ni de } \\
\text { acuerdo, ni } \\
\text { desacuerdo }\end{array}$ & 12 & $18 \%$ \\
\cline { 2 - 4 } & Desacuerdo & 3 & $5 \%$ \\
\cline { 2 - 4 } & $\begin{array}{l}\text { Total } \\
\text { Desacuerdo }\end{array}$ & 0 & $0 \%$ \\
\cline { 2 - 4 } & Total & 65 & $100 \%$ \\
\hline
\end{tabular}

\section{Cuadro 10.- Incremento del arte a nivel nacional e internacional}

Análisis: Los encuestados exteriorizaron su opinión en cuanto a la interrogante de que el aporte que se consiga con los talleres de arte contribuirán a que nuestro país aumente su participación en el ámbito artístico, tanto a nivel nacional como a nivel internacional respecto a esto un 54\% dijo que está totalmente de acuerdo con que así será, el 23\% dijo estar de acuerdo, con un $18 \%$ se manifestaron ni en acuerdo ni desacuerdo frente a esto solo un 5\% comento estar en desacuerdo y ninguno de los encuestados resolvió estar en total desacuerdo con lo planteado en la pregunta. Por lo que se espera obtener excelentes resultados con las capacitaciones dadas a los profesores de la facultad para potenciar las oportunidades en el campo de las artes a nivel nacional. 


\section{Conclusiones.}

Para el 49\% de la población encuestada está de acuerdo con que la integración de las artes puede enriquecer la cultura y fomentarla en la juventud, mientras que el $23 \%$ esta solo de acuerdo, el $5 \%$ se muestra indiferente, un $15 \%$ manifiesta su desacuerdo respecto a esta propuesta planteada y solamente un $8 \%$ está en total desacuerdo con la propuesta. Lo que quiere decir que los docentes consideran en su mayoría que hay factibilidad en esta idea.

Los docentes fueron interrogados respecto al grado de conocimiento que tienen según su apreciación propia y la encuesta arrojo las siguientes respuestas, el $57 \%$ de los encuestados considera que sus conocimientos en el campo de las artes son insuficientes, seguido del $23 \%$ que dice tener poco conocimiento, el 5\% tiene moderados conocimientos y solamente un 15\% manifestó tener los conocimientos que se requieren para impartir la enseñanza en el campo de las artes a los estudiantes.

El 46\% de los docentes considera que la música es una buena opción entre las artes que se puede implementar en la facultad, a esto le sigue con un $22 \%$ Cine, ya que se considera que tendrá un gran aporte profesional para los estudiantes de todas las carreras que existen en la Facso, el 12\% indica que prefiere danza, mientras que el $11 \%$ nombra a pintura como la más idónea y finalmente se ubican con un $3 \%$ arquitectura y con solo un $1 \%$ la escultura siendo el arte que menor grado de aceptación tuvo en la encuesta realizada.

En cuanto a las opiniones acerca de la contribución que representa para los estudiantes la implementación de la educación artística tenemos a un $65 \%$ que se pronuncia totalmente de acuerdo, un $31 \%$ está de acuerdo, el $5 \%$ de los docentes manifiesta que no está de acuerdo ni tampoco en 
Juan C. Loaiza-Mina; Roberto A. Loayza-Mina; Lucrecia de las M. Benítes-Mora; Johnson Y. Ching-Chiy

desacuerdo en este aspecto y finalmente ninguno de los encuestados manifestó estar en desacuerdo ni en total desacuerdo.

Entre los docentes que participaron de la encuesta el 52\% considera que es muy necesario que se realicen talleres de capacitación para los catedráticos en el campo de artes, ya que consideran que tienen falencias en cuanto a conocimientos en esta área, el 29\% cree que es necesario, solo el $19 \%$ muestra una actitud desinteresada y entre los encuestados ninguno piensa que sea poco necesario, ni tampoco creen que la medida es para nada necesaria.

Las disciplinas artísticas en las que destacan interés los maestros encuestados, para los talleres de capacitación son las siguientes: el $26 \%$ prefiere la música, el $22 \%$ el cine, mientras que literatura tiene un $18 \%$ de interés por parte de los docentes, seguida de pintura que abarca un $17 \%$, luego tenemos con un $12 \%$ a danza y con los menores porcentajes de expectativa por parte del personal docente, finalmente se ubican con un $3 \%$ escultura y por ultimo con un $2 \%$ arquitectura.

En cuanto a los horarios a considerarse para la realización de los talleres se les brindo tres opciones, un $15 \%$ manifiesta que de preferencia les gustaría recibir los talleres en la jornada de la mañana, a esto le sigue un $36 \%$ que indica que prefiere que la capacitación sea en jornada nocturna y un $49 \%$ indica que puede asistir en la jornada de la tarde, ya que esta le permite laboral con normalidad y sin interrupciones en su horario de trabajo, cabe destacar que este es el grupo que mayor aceptación presenta en cuanto a la horario de preferencia por parte de los docentes y es el que será considerado para su posterior aplicación.

Los encuestados exteriorizaron su opinión en cuanto a la interrogante de que el aporte que se consiga con los talleres de arte contribuirán a que nuestro país aumente su participación en el ámbito artístico, tanto a nivel nacional como a nivel internacional respecto a esto un 54\% dijo que está 
La cultura artística como parte del perfil del docente, incentivación y desarrollo de las actividades artísticas

totalmente de acuerdo con que así será, el 23\% dijo estar de acuerdo, con un 18\% se manifestaron ni en acuerdo ni desacuerdo frente a esto solo un $5 \%$ comento estar en desacuerdo y ninguno de los encuestados resolvió estar en total desacuerdo con lo planteado en la pregunta. Por lo que se espera obtener excelentes resultados con las capacitaciones dadas a los profesores de la facultad para potenciar las oportunidades en el campo de las artes a nivel nacional.

\section{Bibliografía.}

García Retana, J. (2012). La educación emocional, su importancia en el proceso de aprendizaje. Educación, $36(1), 1-24$.

Mitchel, M. (2009). Ballet point by point. Barcelona: Publising group.

Rico, P. (2009). La Zona de Desarrollo Próximo. Procedimientos y tareas de aprendizaje. La Habana: Pueblo y Educación.

Vázquez, M., Gómez, C., \& Lugo, C. (2008). Historia del arte. México: Cengage Learning.

Yuni, J., \& Urbano, C. (2010). Educación de adultos mayores. Cordoba: Brujas. 